\title{
Kleinräumige Siedlungsanalysen zur Abbildung von Veränderungen: Vom Nutzen baulich-räumlicher Daten für die Planung
}

\author{
Mei-Ing Ruprecht
}

Eingegangen: 31. Juli 2013 / Angenommen: 14. April 2014

(C) Springer-Verlag Berlin Heidelberg 2014

\begin{abstract}
Zusammenfassung Sowohl die bestandsorientierte Siedlungsentwicklung als auch GIS-gestützte baulich-räumliche Analysen werden zukünftig an Bedeutung gewinnen. Es scheint aber eine Wissenslücke zwischen Planern und Geodaten-Bereitstellern bezüglich quantitativer baulichräumlicher Daten zu geben. Der Beitrag behandelt daher den Nutzen und Mehrwert von kleinräumigen baulichräumlichen Daten für die Planung, indem Forschungsarbeiten zur Siedlungsentwicklung, Innenentwicklung und zu Veränderungen am Wohnungsmarkt ausgewertet werden. Es zeigt sich, dass mit baulich-räumlichen Daten Muster und Veränderungen von Siedlungsstrukturen sowie beispielsweise in Verbindung mit Sozialdaten gebäudetypbasierte Bevölkerungsmuster und -veränderungen detaillierter als zuvor beschrieben werden können. Dadurch entstehen wichtige Entscheidungsgrundlagen für die raumbezogene Planung, zur Formulierung und Bewertung von Zielvorgaben sowie für konkrete Maßnahmen. Die amtliche Datenlage ist derzeit jedoch eingeschränkt, so dass für die zukünftige Bereitstellung von baulich-räumlichen Daten Anforderungen formuliert und vorgeschlagen werden. Abschließend wird für eine Verwendung von kleinräumigen baulich-räumlichen Daten im Rahmen von MonitoringSystemen und Geodateninfrastrukturen plädiert, da diese eine wichtige Grundlage für kommunale strategische Entwicklungskonzepte und ein computergestütztes Stadtmanagement darstellen können.
\end{abstract}

Dipl.-Ing. M. Ruprecht $(\bowtie)$

Dresden Leibniz Graduate School, Leibniz-Institut für

ökologische Raumentwicklung e. V.,

Weberplatz 1,

01217 Dresden, Deutschland

E-Mail: m.ruprecht@ioer.de
Schlüsselwörter Gebaute Umwelt · Siedlungsstruktur · Monitoring - Kleinräumige Analysen - Stadtmorphologie · Typisierung

\section{Small-Scale Settlement Analyses for Visualizing Change. Potentials of Using Small-Scale Built Environment Data}

\begin{abstract}
In Germany, a rising relevance of adapting the built environment and a rising relevance of GIS-based analyses of the built environment can be observed. But there seems to be a communication or knowledge lack between planners and providers of built environment data. For this reason, this article investigates potentials for using small-scale built environment data and the additional value of it by conducting a literature review. The focus is on literature concerning settlement development, innercity development and transformation processes of housing. The literature suggests that built environment data opens up effective ways to analyse patterns and transformation of settlement structures as well as population patterns and transformation related to specific housing types. This information can be used for strategic planning, housing policies and evaluation of planning targets. It is also useful for a computer assisted management of cities. As the official data is insufficient and other data sources limited in access, requirements for built environment data are formulated. In conclusion, this article argues for using small-scale built environment data as an essential part of communal monitoring systems and geo data infrastructure.
\end{abstract}

Keywords Built environment - Settlement structure · Monitoring - Small scale analyses · Urban morphology · Typologies 


\section{Ausgangslage}

\subsection{Relevanz kleinräumiger Siedlungsanalysen}

Die heutige Siedlungsentwicklung ist mit vielfältigen Aufgaben konfrontiert - von ökologischen Herausforderungen wie der energetischen Sanierung gealterter Wohnungsbestände oder städtischer Wärmeinseln, sozialräumlicher Polarisierungen und dem demographischen Wandel bis hin zu ökonomischen Herausforderungen wie dem haushälterischen Umgang mit knappen finanziellen Mitteln. Sie werfen die Frage auf, wie Siedlungsstrukturen, insbesondere Wohnungsbestände, aber auch Frei- und Brachflächen, in Zukunft weiterentwickelt werden sollten. Mit dem 30-haZiel der Nationalen Nachhaltigkeitsstrategie hat die Bundesregierung (2002) ein Mengenziel gesetzt, an dem sich die Kommunen orientieren sollen, und hat gleichzeitig mit einer „doppelten Innenentwicklung“ freiraummobilisierende und aufwertende Maßnahmen für eine Qualifizierung des Siedlungsraumes in den Vordergrund gerückt (Hüchtker/Scholz/Selle 2000: 54; Bundesregierung 2002: 68, 291; Siedentop 2010: 236 f.). ${ }^{1}$ Damit verschiebt sich der Schwerpunkt von der Außenentwicklung zur Innenentwicklung, das heißt von einer wachsenden Siedlungsentwicklung auf eine bestandsorientierte Weiterentwicklung von Siedlungsstrukturen. Maßnahmen sind die Erneuerung und gegebenenfalls Nachverdichtung der bestehenden Siedlungsstrukturen, die Umnutzung brachliegender Militär-, Gewerbe- oder Bahnareale oder die (gelenkte) Entdichtung von Siedlungsstrukturen und Schaffung neuer Freiflächen bei strukturellem Wohnungsleerstand.

Parallel dazu gewinnen räumliche Analysen mit GIS, Monitoring-Systemen und ein computergestütztes, intelligentes' Management von Städten an Bedeutung. In zahlreichen Städten steht ein sozialräumliches Monitoring im Vordergrund, um die allgemeine soziale Entwicklung der städtischen Teilräume zu beobachten, soziale Problemlagen zu identifizieren oder die soziale Infrastruktur zu planen (vgl. Häußermann/Dohnke/Seidel-Schulze 2010: 3). Die amtliche Datenlage lässt jedoch nur in seltenen Fällen eine kleinräumige Verknüpfung von baulich-räumlichen und sozialräumlichen Indikatoren zu. Übergreifende Analysen zu ausgewählten Bautypen und Bauperioden sind nicht möglich (BMVBS/BBSR 2009: 139). Eine Schwierigkeit einer Vielzahl von quantitativen Arbeiten zur Beobachtung von bestimmten Wohnungsbeständen bzw. Gebäudetypen liegt darin, dass es innerhalb eines statistischen Raumbezugs meist keine homogenen Gebäudetypen gibt, das heißt, dass

\footnotetext{
${ }^{1}$ Mit der „doppelten Innenentwicklung“ ist nach Hüchtker/Scholz/ Selle (2000: 54) die „doppelte Nutzungschance“ gemeint, brach gefallene Areale für Siedlungsnutzungen zu reaktivieren, aber auch neue Freiräume in der Stadt entstehen zu lassen.
}

es sich meist um Mischgebiete, z. B. aus Einfamilienhäusern und Zeilenbauten, handelt. Einen alternativen Ansatz bieten zwar sogenannte Indikatorgebiete, die typische Entwicklungen repräsentieren sollen (Bizer/Ewen/Knieling et al. 2010: 114, 124), diese sind aber, wenn sie Stadtteile als statistischen Raumbezug verwenden, sehr grobkörnig und Erkenntnisse daraus sind, wie auch aus qualitativen Quartiersanalysen, nur bedingt auf andere Gebiete übertragbar. Unabhängig davon, welche Problemstellung anvisiert wird, fehlt den Kommunen eine kleinräumige baulich-räumliche Datengrundlage, um statische Daten zur Bevölkerung in Siedlungsstrukturen zu ,verankern'. Es scheint eine massive Wissenslücke oder ,Wissens-Asymmetrie` zwischen den Entwicklern von Dateninfrastruktur und den Nutzern (z. B. Planungsämtern) zu geben und den Kommunen scheint beim Aufbau einer Geodateninfrastruktur ein Bezug zu übergeordneten (lokal)politischen Zielsetzungen bzw. zu einer nachhaltigen Stadtentwicklung zu fehlen (Wolfram 2011: 245 f.)

Kazig und Wiegandt (2006) sehen einen Grund dafür, dass die Beschreibung der gebauten Umwelt an Bedeutung verloren hat, in dem „Abschied vom Landschaftsparadigma als zentraler Perspektive geographischen Denkens Ende der 1960er Jahre“. Ihnen zufolge befinden wir uns aber in einer Phase der Neuthematisierung der gebauten Umwelt. Frick (2011: 16) unterscheidet vier Sichtweisen auf den Gegenstand „Stadt“, nämlich

- die Stadt als Gesellschaft bzw. deren soziale Entwicklung,

- die Stadt als Markt bzw. deren wirtschaftliche Entwicklung,

- die Stadt als Biotop bzw. deren ökologische Entwicklung und

- die Stadt als „Bauwerk“ bzw. deren baulich-räumliche Entwicklung.

Folgt man dieser Argumentation, so lässt sich schließen, dass die Entwicklung der baulich-räumlichen Siedlungsstruktur eine sehr hohe Bedeutung für die Entwicklung der anderen drei Bereiche, also Gesellschaft, Markt und Biotop hat. Sofern man die Qualifizierung des Siedlungsraumes ernst nimmt, wird man folglich genauer als bisher baulich-räumliche Strukturen und Entwicklungen und deren Zusammenhang mit sozialen und ökologischen Entwicklungen analysieren müssen, um Entscheidungsgrundlagen für Zielvorgaben und konkrete Planungen zu schaffen.

Die Relevanz kleinräumiger Siedlungsanalysen basiert demnach darauf, dass sowohl eine bestandsorientierte Siedlungsentwicklung als auch GIS-gestützte baulich-räumliche Analysen für die Planung an Bedeutung gewinnen. Das Problem liegt darin, dass eine Wissenslücke zwischen Planern und Geodatenanbietern besteht bezüglich dem Mehrwert quantitativ auswertbarer baulich-räumlicher Daten und den Anforderungen der Planung an die Daten. 


\subsection{Begriffe}

Mit baulich-räumlichen Daten sind Daten gemeint, die Merkmale der baulich-räumlichen Organisation von Siedlungsstrukturen beschreiben. Im weiteren Sinne ist nach Cerdà ${ }^{2}$ mit der baulich-räumlichen Organisation von Gebäuden und Grundstücken ihre Art und Weise der Anordnung und ihre Beziehung zueinander gemeint (Frick 2011: 17). Im engeren Sinne sind baulich-räumliche Daten quantitativ verfügbare Geodaten, z. B. zur Gebäudegeometrie, zu Gebäudetypen, Bauperiode und Bebauungsdichte. Unter Stadtstrukturtypen werden Kategorien verstanden, die ähnliche Merkmale hinsichtlich Gebäudetyp, Bebauungsform, Dichte, Bauperiode (Materialität, Konstruktion) oder Freiflächen aufweisen. Der Strukturtypenansatz ist ein methodischer Ansatz, bei dem Stadtstrukturtypen auf unterschiedlichen Maßstabsebenen je nach Zielsetzung der Forschungsarbeit gebildet werden (vgl. Arlt/Blum/Gruhler et al. 2010: 28).

\subsection{Ziel des Beitrags}

Der Beitrag widmet sich der Frage, welchen Nutzen und Mehrwert baulich-räumliche Daten für die Planung und insbesondere die bestandsorientierte Siedlungsentwicklung darstellen können. Daraus ergeben sich die Fragen, welche Anwendungsbereiche es für baulich-räumliche Daten gibt, welche Daten und Methoden dabei genutzt werden, wie das derzeitige Angebot an baulich-räumlichen Daten ist und welche Anforderungen es an diese Daten gibt. Aus der Gegenüberstellung von Bedarfen der Forschungsarbeiten mit dem derzeit verfügbaren Angebot an baulich-räumlichen Daten werden sodann die Anforderungen an die Daten bzw. die Datenlage abgeleitet.

Diesem Beitrag liegt die These zugrunde, dass kleinräumig verfügbare baulich-räumliche Daten derzeit eine zu geringe Bedeutung in der Planung einnehmen, aber darin ein hohes Potenzial liegt als Grundlage für Entscheidungen zu städtebaulichen und baulichen Maßnahmen und damit auch sozialen, ökologischen und ökonomischen Fragen. Ziel des Beitrags ist, die genannte Wissenslücke zwischen Planern und Geodatenanbietern zu überbrücken. Der Beitrag richtet sich damit sowohl an Kommunen, insbesondere an Akteure in den Statistikstellen und den Stadtplanungsämtern, als auch an Wissenschaftler und Praktiker, die an kleinräumigen Siedlungsanalysen interessiert sind.

\subsection{Auswahl der Forschungsarbeiten}

Im Rahmen einer Literaturübersicht von wissenschaftlichen Analysen aus Städtebau, Geographie und Kartographie wer-

\footnotetext{
${ }^{2}$ Ildefons Cerdà, ein katalanischer Stadtplaner, plante 1859 die Erweiterung Barcelonas.
}

den Forschungsarbeiten ausgewählt, in denen baulich-räumliche Daten für planungsrelevante Analysen und Aussagen verwendet wurden und deren Zielsetzung und Ergebnisse einen besonderen Nutzen und Mehrwert von baulichräumlichen Daten zeigen. Es wurden vorwiegend Studien aus dem deutschsprachigen Raum ausgewählt und diese um internationale Studien ergänzt, wenn dadurch weitere Anwendungsbereiche aufgezeigt werden konnten. Dabei wurden sowohl ältere als auch aktuelle Arbeiten berücksichtigt, um ein Spektrum der Nutzung baulich-räumlicher Daten zu erhalten. Die Durchsicht der deutschsprachigen Literatur zeigte drei Schwerpunkte, die für die Planung eine hohe Relevanz haben:

- Abbildung der Siedlungsentwicklung

- Gestaltung, Beobachtung und Bewertung der Innenentwicklung

- Kleinräumige Beobachtung von Wohnungsbeständen

Daher werden die Arbeiten nach diesen drei Themen gruppiert betrachtet.

\section{Anwendungsbereiche baulich-räumlicher Daten}

\subsection{Abbildung der Siedlungsentwicklung}

Die Forschungslinie, die traditionell mit der Abbildung und Analyse von baulich-räumlichen Daten arbeitet, ist die Stadtmorphologie. Die Stadtmorphologie bezeichnet die zwei- und dreidimensionale Gestalt im Sinne der baulichphysischen Struktur der Stadt (Curdes 1993: 63). Sie hat Wurzeln in Deutschland, Großbritannien und Italien (vgl. Whitehand 1988; Curdes 1989; Heineberg 2006). Ziel der stadtmorphologischen Analyse ist, die baulich-räumlichen Eigenschaften und Veränderungen der Siedlungsstruktur im Wandel der Zeit zu beschreiben.

Im Themenheft „Urban Morphology“ der Zeitschrift „Urban Design“ (2005) beschreiben internationale Vertreter der Stadtmorphologie überblicksartig die Relevanz der Stadtmorphologie für die Stadtgestaltung. Grundsätzlich geht es darum, die Strukturen der Stadtgestalt zu entdecken und deren Veränderung zu beobachten, um mit dieser historisch-geographischen Langzeitbeobachtung eine Entscheidungsgrundlage für Planer, Architekten, Städtebauer und Politiker zu schaffen (Evans 2005: 16; Whitehand 2005: 20). Für Whitehand (2005: 20) ist die „urban landscape unit“ ein wichtiges Instrument, um Gebiete mit einem eigenständigen Charakter abzugrenzen. Diese stellten eine wichtige Basis für die Analyse der Stadt- bzw. Landschaftsgestalt und deren Gestaltung dar. Auch Larkham (2005: 24) spricht sich für diese ,character areas“ aus, um die vorhandenen guten Qualitäten der Stadtgestalt zu identifizieren, zu erhalten oder zu verstärken und um dieses Wissen zur Kommunikation mit 
Eigentümern zu nutzen. Diese Erkenntnisse basieren auch auf Studien von Conzen, Protagonist der Stadtmorphologie in Großbritannien, der sich bereits in den 1960er Jahren für ein „townscape management" zur Entwicklung und Pflege der Stadtgestalt mithilfe der Stadtmorphologie aussprach (Conzen 1960; Conzen 2004: 57 f.).

Ein gutes Beispiel für die Abbildung der Siedlungsentwicklung ist das Werk „Stadtstruktur und Stadtgestaltung“ von Curdes (1993). Für die Analyse der Siedlungsentwicklung wurden Gebäudestrukturen, Hauptverkehrsnetze und auch Bahnflächen mithilfe von Karten beschrieben und unterschiedliche prägnante Zeitstände zwischen 1860 und 1980 verglichen. Ziel dieser stadtmorphologischen Analysen war es, baulich-räumliche Strukturen (Muster, Regelmäßigkeiten, Siedlungs- und Stadtstrukturtypen) sowie bauliche Veränderungen zu entdecken, zu beschreiben und zu bewerten sowie im Weiteren Hinweise für Innovationen zu erhalten. Ergebnis der Studien ist eine sehr anschauliche Darstellung in Schwarzplänen (nur Gebäude) und Netzplänen (nur Straßen/Verbindungen), die Dichte, Schwerpunkte und Brüche der baulichen Entwicklungen sowohl räumlich als auch inhaltlich verdeutlichen (Curdes 1993: 35 ff.). ${ }^{3}$

In einer aufwendigen Studie zu „Wohnungsbau und Siedlungsentwicklung in Dortmund“" erfasste Kühne (1983: 55) alle städtebaulich „relevanten“ Siedlungen Dortmunds mit dem Ziel, einen Überblick über die unübersichtliche Siedlungsentwicklung bzw. „Zersiedelung“ der Stadt zu bekommen. Er erhob dazu die Quartiere nach ihren Erbauungsdaten, ihren räumlichen Abgrenzungen sowie Standortmerkmalen und nutzte kartographische Darstellungen für die Visualisierung der Daten. Abschließend wurden die Quartiere vor dem Hintergrund der historischen Entwicklung und des damalig aktuellen Erscheinungsbilds in Gebietstypen zusammengefasst, um Hinweise auf die Standortqualität zu erhalten (Kühne 1983: 71).

Eine neuere Arbeit ist „Schichten einer Region - Kartenstücke zur räumlichen Struktur des Ruhrgebiets“" (Reicher/ Kunzmann/Polivka et al. 2011), die von „Die Schweiz - Ein städtebauliches Portrait" (Diener/Herzog/Meili et al. 2006) inspiriert wurde. Ziel des Ruhrgebietbuches war, die spezifischen Eigenheiten des Ruhrgebiets aufzuzeigen, um daraus eine räumliche und städtebauliche Vision zu entwickeln. Eine der sieben regionalen Schichten bilden die Karten der Siedlungs- und Bebauungsstruktur, durch deren Darstellung sowohl die enorme Siedlungsentwicklung seit 1840 als auch die Verteilungsmuster von kompakten und lockeren Bebauungstypen, Industrie- und Gewerbe- sowie Freiflächen gezeigt werden. Hinzu kommen Analysen von ,inneren Rändern“, bei denen Siedlungs- und Freiräume sich begeg-

\footnotetext{
${ }^{3}$ Die detaillierte Darstellung der Entwicklung von Stadträumen findet sich für die Städte Aachen in Curdes (1999) und Köln in Curdes/Ulrich (1997).
}

nen, und „Zäsuren“, die als Gewässer, Straße, Bahnschiene oder autonome Industriefläche die Landschaft und den Siedlungsraum gliedern und prägen (Polivka/Roost 2011: 41 ff., 57, 66 f.). Der Blick wird in dieser Analyseschicht nicht nur auf die Abbildung der Siedlungsentwicklung, sondern auch auf die Anordnung und Typen bzw. Regelmäßigkeiten von baulich-räumlichen Strukturen gelenkt.

Eine der ersten Arbeiten, die sich gezielt und systematisch mit strukturellen Gesichtspunkten beim Stadtumbau schrumpfender Städte, insbesondere in Ostdeutschland, auseinandersetzt, ist der Sammelband „Stadtlichtungen - Irritationen, Perspektiven, Strategien“" (Giseke/Spiegel 2007b). Die Herausgeberinnen riefen dazu auf, Konzepte für baulich-räumliche Strukturen zu entwickeln, um bei Abrissen und den Auswirkungen des Bevölkerungsrückganges nicht nur bautechnische, funktionale und wirtschaftliche Gründe anzuführen (Giseke/Spiegel 2007b: 7, 12). Ein Beitrag erörtert, ob Strukturmodelle der Wachstumslenkung auch zur Schrumpfungslenkung geeignet sind (Albers 2007), während ein anderer die „Stadtverdünnung“ unter baulichräumlichen Merkmalen darstellt (Jessen 2007). ${ }^{4}$

Ein großes methodisches Potenzial zeigt sich in der Verarbeitung kartographischer Daten in GIS. Methodisch und technisch ist daher eine Arbeit von besonderem Interesse, in der Dießelmann/Meinel/Hecht (2011) ein computergestütztes Vorgehen zum Siedlungsflächenmonitoring in Rasterkarten vorschlagen. Dabei werden multitemporale Daten zur Flächennutzung in einem Raster ausgewertet. Für jeden Zeitschnitt wird zwischen Siedlungs- und NichtSiedlungsflächen unterschieden. Für ein Monitoring der Siedlungsflächenentwicklung werden sodann die Rasterkarten unterschiedlicher Zeitschnitte verglichen (Dießelmann/Meinel/Hecht 2011: 67). In Vorarbeiten dazu wird eine prozesshafte Darstellung aus der Überlagerung von Karten eines Gebiets vorgestellt, durch die die quantitative Beschreibung der baulichen Veränderungen bis auf die Gebäudeebene möglich wird (vgl. Meinel/Hecht/Herold et al. 2008). Neubauten und Abrisse (aber auch Brachflächen) können auf diese Weise im Siedlungsgefüge erkannt und im Abgleich der Karten die Umsetzung der flächenpolitischen Ziele der Nationalen Nachhaltigkeitsstrategie bilanziert werden (Meinel/Hecht/Herold et al. 2008: 62).

Die vorgestellten Arbeiten zielen auf eine Abbildung der baulich-räumlichen Struktur und Entwicklung, um Hinweise zu Charakteristiken, zur Erhaltung und zur Weiterentwicklung der Stadtgestalt zu ermitteln. Als wesentliche Methode wurde der kartographische Vergleich verwendet (vgl. Abb. 1). Die Arbeiten zeigen die Bedeutung der

\footnotetext{
${ }^{4}$ Als Forschungslücke werden systematische Untersuchungen von Stadtschrumpfung hinsichtlich Strukturmodellen in einer Art ,negativer urbaner Morphologie“ genannt (Jessen 2007: 54 f.). Konzeptionelle Strukturmodelle des Schrumpfens wurden beispielsweise vom „Büro für urbane Projekte“ aus Leipzig entwickelt.
} 
Abb. 1 Siedlungsentwicklung am Beispiel Hannover (Groß Buchholz) mit identifizierten Wohngebieten der 1950er/1960er Jahre. (Quelle: Geodaten: SEMENTA ${ }^{\circledR}$; Basis-DLM (C); GeoBasis-DE (BKG 2013); Karten: eigene Darstellung)

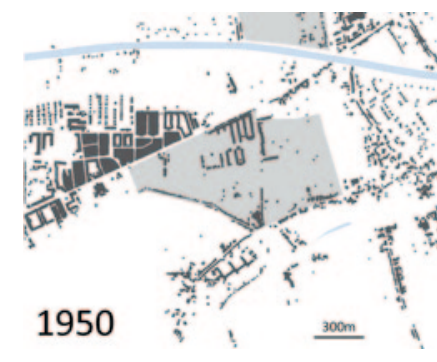

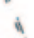

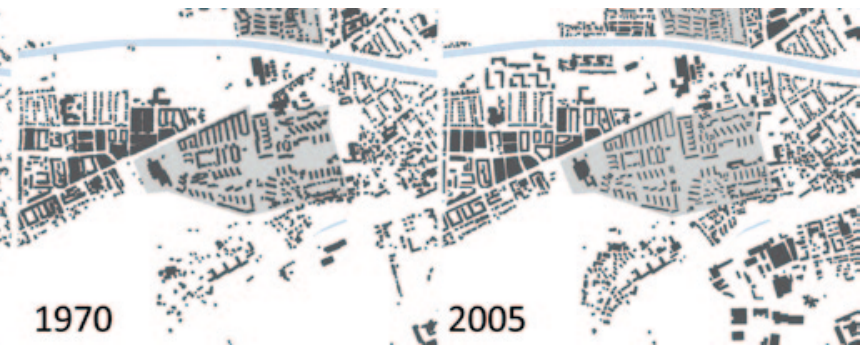

Methode des kartographischen Vergleichs, um sowohl Siedlungserweiterungen als auch Nachverdichtungen, Entdichtung und Umbau in den bestehenden Siedlungsstrukturen abzubilden, zu interpretieren und mit zunehmender Relevanz auch zu quantifizieren. Da zahlreiche Einzelabrisse zu einer langsamen Fragmentierung des städtischen Gefüges führen können, kann der kartographische Vergleich im Hinblick auf die Gesamtstadt bei der Einschätzung helfen, ob langfristig stadtbildprägende und gegebenenfalls identitätsstiftende baulich-räumliche Strukturen verloren gehen. Dies gilt analog für Neubauten und Verdichtung in wachsenden Städten verbunden jeweils mit der Frage, in welche Richtung sich die Siedlungsstruktur entwickeln soll. Die Abbildungen der Siedlungsentwicklung können damit als Entscheidungsgrundlage für die Formulierung von Zielvorgaben für die städtebauliche Gestaltung und städtebauliche Maßnahmen genutzt werden. Die Visualisierung unterschiedlicher Merkmale von Siedlungsstrukturen kann ein wichtiges Kommunikationswerkzeug für Planende und die Öffentlichkeit darstellen (vgl. Moudon 1998).

\subsection{Gestaltung, Beobachtung und Bewertung der Innenentwicklung}

Bereits 1983 wird die Innenentwicklung der Städte betont (Bundesministerium für Raumordnung, Bauwesen und Städtebau 1986: 15). Lütke-Daldrup (1990) untersuchte in diesem Zusammenhang die Möglichkeiten, Auswirkungen und Grenzen der Innenentwicklung im Rahmen eines bestandsorientierten Städtebaus und führte dazu eine Pilotstudie im Gebiet des Umlandverbandes Frankfurt durch. Ausgangsthese und Forschungsansatz war, dass man bei Innenentwicklungspotenzialen vom Grundstück und den Veränderungsbedürfnissen der Eigentümer bzw. Nutzer ausgehen müsse, anstatt von einer block- oder quartiersbezogenen Strukturanalyse, wobei der parzellenscharfe Mikroansatz in Wechselbeziehung zu den stadtstrukturellen Gegebenheiten stehen würde (Lütke-Daldrup 1990: 69). Dafür wurden zunächst homogene Siedlungstypen hinsichtlich Bebauungsdichte, Gebäudealter und Gebäudetypen sowie Freiflächenanteile gebildet und die tatsächlichen (nicht nur die theoretischen) Innenentwicklungspotenziale für 15 Modellgebiete erhoben. Die Erkenntnisse mündeten in einer Hochrechnung der Innenentwicklungspotenzi- ale und einer Maßnahmentypologie zur Innenentwicklung (Lütke-Daldrup 1990: 249 f.).

Koll-Schretzenmayr/Ritterhoff/Siebel (2009) und KollSchretzenmayr/Kramp (2010) führten Studien zur Reurbanisierung und baulichen Dynamik in Zürich durch. ${ }^{5}$ Im Mittelpunkt stand die Frage, ob, in welchem Umfang und wo in Zürich Reurbanisierung zu beobachten sei und mit welchen sozialen und sozial-räumlichen Polarisierungen dies verbunden wäre. Dazu verwendeten die Autoren adressgenaue Daten zur Neubautätigkeit, zu Renovationen und Abrissen, die als statistische Daten der Stadt Zürich geführt werden, ${ }^{6}$ sowie sozioökonomische Daten zur Bevölkerung. Ergebnis hinsichtlich der Stadtgestalt ist, dass zwischen 1994 und 2007 eine Vielzahl kleinteiliger Abrisse und Ersatzneubauten vorgenommen wurden, durch die sich der umbaute Rauminhalt verdoppelt und die Anzahl der Wohnungen sogar vervierfacht hate. Parallel dazu war eine sozioökonomische Aufwertung der Bewohnerschaft zu beobachten. Eine derartige innere Verdichtung mit einem strukturellen Umbau des Gebäudebestands führe zu einer tiefgreifenden Veränderung der städtebaulichen Struktur - einem Phänomen, von dem die Kommune aus Sicht der Autoren möglicherweise ,überrumpelt" wurde (KollSchretzenmayr/Kramp 2010: 74). Die Autoren plädieren dafür, neben den sozialen und sozioökonomischen Auswirkungen auf die Bevölkerungsstruktur auch auf die Entwicklung einer Quartiersqualität zu achten, bei der über die Parzellengrenze hinaus gedacht wird (Koll-Schretzenmayr/ Kramp 2010: 77).

Mit ihrer Publikation „Dichter“ dokumentierte die Stadt Zürich architektonische Beispiele von baulichen Veränderungen in der Stadt und reflektierte damit die zunehmende Verdichtung. Im Zuge einer räumlichen Entwicklungsstrategie wurden dem gesamten Siedlungsgebiet anvisierte

\footnotetext{
${ }^{5}$ Hier werden zwei Schweizer Forschungsarbeiten vorgestellt, da ähnliche Arbeiten zu deutschen Kommunen nicht vorliegen und diese zwei Beispiele sehr gut zeigen, wie die Innenentwicklung in Deutschland zukünftig beobachtet und evaluiert werden könnte.

${ }^{6}$ Die Stadt Zürich hat sehr kleinräumige Daten zu baulichen Veränderungen. Mit den Renovationen werden alle baulichen Veränderungen erfasst, die genehmigungspflichtig sind. Da die Stadt Zürich bauliche Veränderungen sehr streng kontrolliert, fallen illegale bzw. ungenehmigte Veränderungen nicht ins Gewicht (Koll-Schretzenmayr/Ritterhoff/Siebel 2009: 2745).
} 
Dichtegrade zugeordnet. Für die Abschätzung der städtebaulichen Charakteristiken und Verdichtungspotenziale wurden die bestehenden Stadtstrukturen in Kernstadt, Dorfkerne, Blockrandgebiete, Transformationsgebiete, urbane Wohnquartiere, offene Baustrukturen, Einfamilienhausund Villengebiete unterschieden (Amt für Städtebau Zürich 2012: 13, 16 f.).

In Deutschland liegt bisher aufgrund der flächenpolitischen Ziele der Nationalen Nachhaltigkeitsstrategie von 2002 ein Schwerpunkt auf und ein großer Bedarf an Informationen zu Innenentwicklungspotenzialen. Daher wird derzeit eine Studie im Auftrag des Bundesinstituts für Bau-, Stadt- und Raumforschung durchgeführt (BBSR 2011), in der mithilfe von baulich-räumlichen Daten bundesweit Innenentwicklungspotenziale entdeckt und quantifiziert werden sollen. Im Fokus der Analyse stehen insbesondere Umfang, Struktur und räumliche Verteilung von Innenentwicklungspotenzialen, mögliche Mobilisierungshemmnisse sowie praktische Erfahrungen der Kommunen mit Flächeninformations- und Managementsystemen.

Die vorgestellten Forschungsarbeiten zeigen, wie die Innenentwicklungspotenziale mithilfe von Karten, qualitativen Vorarbeiten und Stadtstrukturtypen geschätzt werden können. Zudem konnte mit den Beispielen aus Zürich gezeigt werden, wie sozialräumliche Veränderungen durch bauliche Verdichtung beobachtet und qualitative Entscheidungen zur Innenentwicklung mithilfe von Dichtekarten und Siedlungstypisierungen (als Teil von Strategieplänen) unterstützt werden können und wie hilfreich baulich-räumliche Daten für die Gestaltung, Beobachtung und Bewertung der Innenentwicklung sind.

\subsection{Monitoring der Wohnungsbestände}

Das Bundesinstitut für Bau-, Stadt- und Raumforschung ließ 2009 in einer Studie zu den Wohnungsbeständen der 1960er/1970er Jahre den Status quo und die Entwicklungsperspektiven dieser Bestände untersuchen, um problematischen Entwicklungen durch „Strategien für Wohnstandorte an der Peripherie der Städte und in Umlandgemeinden“ (BMVBS/BBSR 2009) frühzeitig zu begegnen. Gegenstand der Forschung waren nicht die ,typischen ' Großsiedlungen, sondern gerade nicht so bekannte homogene und gemischte Bestände aus Geschosswohnungsbau und Einfamilienhäusern, die mithilfe einer Quartiersdatenbank der Firma empirica und telefonischer Interviews ermittelt wurden. Das inhaltliche Ergebnis der Studie war, dass unterschiedliche Entwicklungsstrategien je nach Rahmenbedingungen der Quartiere erforderlich sind und zukünftig eine kommunale Lenkung als notwendig erachtet wird. Die Autoren empfehlen, Konzeptentwicklungen anzustoßen, die zeigen, wie unterschiedliche Gebäudetypen bzw. Stadtstrukturen baulich-räumlich und energetisch ausgebaut und wie übertragbare Ergebnisse erreicht werden können. Die methodische Erkenntnis war, dass es kleinräumigere Daten zu Gebäudetypen und Bauperioden geben sollte, um gebäudetypbasierte Entwicklungen besser analysieren zu können (BMVBS/BBSR 2009: 136, 139, 141).

Der Sammelband „Typologien der gebauten Umwelt“ vereint Forschungsarbeiten, die auf der Grundlage von Stadtstrukturtypen Muster und Veränderungen der gebauten Umwelt untersuchen (Blum/Gruhler 2010). Die Anwendungsbeispiele der Stadtstrukturtypen finden sich auf regionaler, städtischer und teilstädtischer Ebene und verbinden baulich-räumliche Daten mit ökologischen, wirtschaftlichen und sozialen Fragestellungen, z. B. hinsichtlich Bodenversiegelung, Freiraumpotenzialen, Infrastrukturkosten und Entwicklung von Wohnungsteilmärkten (Arlt/ Blum/Gruhler et al. 2010: 31, 33). Banse und Effenberger bildeten für Dresden 13 Stadtstrukturtypen auf der Ebene der statistischen Bezirke. Unterschieden wurden Ein- und Zweifamilienhausgebiete (aller Bauperioden) sowie Mehrfamilienhausgebiete als ,reine Gebiete“ und Mischgebiete nach jeweils sechs Bauperioden. Diese wurden wiederum nach drei Wohnlagen differenziert. Im Ergebnis konnten deutliche Unterschiede in den Veränderungen der "reinen“ und der Mischgebiete sowie nach den Bauperioden festgestellt werden, wobei sich insbesondere in den "reinen“ Gebieten bestimmter Bauperioden Probleme der Überalterung, Sozialstruktur und Leerstand anbahnten (Banse/ Effenberger 2010: 96, 107). Die anderen Beiträge des Sammelbandes basierten ebenfalls auf Stadtstrukturtypen, die zumeist hinsichtlich Gebäudetyp, Bauperiode und Geschossflächendichte gebildet wurden.

In ähnlicher Weise wie in den vorgenannten Arbeiten wurden mit „Complexity and contradiction in ageing early postwar suburbs of Québec City“ baulich-räumliche Daten mit Umfeld- und Verhaltensstudien der Bewohner sowie Elementen der Stadtgestaltung überlagert (Vachon/Luka/Lacroix 2004). Mit der morphologischen Analyse konnten Schlüsselelemente des gebauten Raumes, Nutzungsmuster, Verkehr und auch Bedeutungen erkannt werden. Ein wesentlicher Erkenntnisgewinn liegt in der Typenbildung der Vororte. Weiterer Forschungsbedarf wurde in der Anreicherung der morphologischen Analyse mit demographischen und sozioökonomischen Merkmalen sowie zur sozialen Lage gesehen.

Mit einem Nutzungszyklus-Management zeigten Bizer/ Ewen/Knieling et al. (2009; 2010) auf, wie durch ein Monitoring von Stadtquartieren Qualitäten entwickelt und Flächenverbrauch gemindert werden kann. Die Grundlage bildeten homogene Gebietstypen, die flächendeckend für eine gesamte Stadt abgegrenzt werden sollten (Bizer/Ewen/ Knieling et al. 2010: 114, 124). In einem ersten Schritt eines Nutzungszyklus-Managements wurden Indikatorgebiete bzw. Beobachtungsgebiete mit den drei Hauptindikatoren Bauperiode, Anteil der über 65-Jährigen und der Leis- 
tungsempfänger nach SGB II ausgewählt. Daraufhin soll ein vertieftes Monitoring von problematischen Gebieten mittels 14 Indikatoren stattfinden, wobei die Themenfelder „soziodemographischer Lebenszyklus“, „Nachfrageentwicklung im Quartier“ und der „,baulich-räumliche Zyklus“ (Gebäudealterung) in den Vordergrund gestellt werden. Aus der vertiefenden quantitativen Analyse kombiniert mit einer qualitativen Befragung könnten sodann Maßnahmen zu Wohnung, Wohnumfeld, Versorgung und Nachbarschaft abgeleitet werden (Bizer/Ewen/Knieling et al. 2009: 28, 30).

Aufgrund der eingeschränkten kleinräumigen Datenlage zu baulich-räumlichen Merkmalen gibt es erst wenige Beispiele für die Verknüpfung von sozialen und baulich-räumlichen Daten. Aus den vorgestellten Forschungsarbeiten lässt sich für das Monitoring von Wohnungsbeständen aber ableiten, dass durch gebäudetypbasierte Beobachtungen für die Weiterentwicklung der Wohnungsbestände belastbare und genauere Grundlagen für die involvierten Akteure möglich werden. Zudem können Typisierungen bzw. Typologien von städtischen, aber auch suburbanen oder dörflichen Strukturen bei Einschätzungen der Quartiersentwicklung helfen und eine höhere Differenzierung der Problemlagen zulassen.

\subsection{Zwischenfazit zu den Anwendungsbereichen}

Die Literaturbeispiele zu den drei Themen der wachsenden Siedlungsentwicklung, der Innenentwicklung und der Entwicklung der Wohnungsbestände zeigen ein breites Spektrum an Anwendungsbereichen baulich-räumlicher Daten. Für die Siedlungsentwicklung helfen baulich-räumliche Daten, die Neubautätigkeit und gegebenenfalls Abrisstätigkeit zu quantifizieren und zu lokalisieren. Damit können Veränderungen der Siedlungsstruktur erkannt sowie die bisherige und zukünftige Ver- und Entdichtung des Bestands eingeschätzt werden. Die Quantifizierung dient der Evaluierung von Zielvorgaben der Nationalen Nachhaltigkeitsstrategie der Bundesregierung oder beispielsweise auch Stadtentwicklungskonzepten. Kleinräumige baulich-räumliche Daten können ebenso dazu beitragen, Innenentwicklungspotenziale zu lokalisieren und zu quantifizieren. Mit einer kleinräumigen Wohnungsmarktbeobachtung können weiterhin nicht nur Stadtteile, die von einer bestimmten Bauperiode dominiert werden, sondern auch gebäudetypbasierte Analysen durchgeführt werden. Solche Analysen mittels Stadtstrukturtypen, bei denen baulichräumliche Daten beispielsweise mit sozialen Daten verknüpft werden, können Problemlagen differenziert aufzeigen und zu konkreten, räumlich verorteten Handlungsempfehlungen für ausgewählte Gebäudetypen führen.

Die aufgeführten Siedlungsanalysen lassen sich in vier Felder untergliedern (vgl. Tab. 1). Auf der einen Seite stehen Analysen, die sich nur auf baulich-räumliche Merkmale stützen, sei es um Verteilungsmuster und Regelmäßigkeiten in der baulich-räumlichen Struktur zu erkennen oder sei es,
Tab. 1 Untergliederung kleinräumiger Siedlungsanalysen

\begin{tabular}{lll}
\hline Gegenstand & $\begin{array}{l}\text { Baulich-räumliche } \\
\text { Merkmale }\end{array}$ & $\begin{array}{l}\text { Baulich-räumliche und } \\
\text { soziale Merkmale }\end{array}$ \\
\hline $\begin{array}{l}\text { Muster/ } \\
\text { Struktur }\end{array}$ & $\begin{array}{l}\text { Anzahl, Anordnung und } \\
\text { Verteilung von Gebäuden } \\
\text { und Stadtstrukturtypen }\end{array}$ & $\begin{array}{l}\text { Sozialräumliche Ver- } \\
\text { teilung der Bevölkerung } \\
\text { in Bezug auf Gebäude- } \\
\text { bzw. Stadtstrukturtypen }\end{array}$ \\
Entwicklung & $\begin{array}{l}\text { Bauliche Veränderungen } \\
\text { durch Neubau, Umbau, } \\
\text { Ersatzneubau und Abriss }\end{array}$ & $\begin{array}{l}\text { Veränderung der Be- } \\
\text { venhang in Zusam- } \\
\text { menhit baulichen } \\
\text { Veränderungen }\end{array}$ \\
\hline
\end{tabular}

um Veränderungen der Siedlungsstruktur zu beschreiben (zumeist ,reine“ stadtmorphologische Analysen). Auf der anderen Seite stehen Analysen, in denen baulich-räumliche Daten mit Sozialdaten verknüpft werden, um sowohl Bevölkerungsmuster für bestimmte Stadtstrukturtypen (z. B. soziale Probleme oder Überalterung der Bewohnerschaft in bestimmten Wohnungsbeständen) als auch Veränderungen der Bevölkerung aufgrund von baulichen Veränderungen (z. B. sozioökonomische Aufwertung bei baulicher Verdichtung) zu erkennen. ${ }^{7}$ Methodisch basieren diese einerseits auf kartographischen Analysen und andererseits auf statistischen Analysen wie Cluster- und Regressionsanalysen.

\section{Angebot an baulich-räumlichen Daten}

\subsection{Amtliche Statistik und kartographische Daten}

Die baulich-räumlichen Daten des Zensus 2011 beinhalten deutschlandweit auf Gemeindeebene die Indikatoren Bauperiode (Jahrzehnte, Jahrzwanzigste), Gebäudetyp (freistehendes Haus, Doppelhaushälfte, gereihtes Haus, anderer Gebäudetyp) und die Wohnungszahl. ${ }^{8}$ Die Gebäude- und Wohnungsdaten im Zensus wurden auch unterhalb der Gemeindeebene beispielsweise für Stadtteile und für Straßenzüge erfasst, ebenso wie Daten zum baulichen Zustand. Die neuen Daten lassen bisher trotz der detaillierten Erhebung nur eine Auswertung auf der Ebene der Gemeinden zu. Die Melderegister der Kommunen dürfen mit den Zensusdaten nicht verbessert werden. Die Frage eines Zuganges zu anonymisierten Primärdaten über Forschungsdatenzentren ist derzeit noch in Klärung. ${ }^{9}$

\footnotetext{
${ }^{7}$ Ähnlich lassen sich baulich-räumliche Merkmale mit ökologischen Merkmalen verknüpfen. Der Stadtstrukturtypenansatz hat eine lange Tradition in der stadtökologischen Forschung (vgl. Schiller 2010: 75). Beispielsweise können über Stadtstrukturtypen die Bodenversiegelung, Grünausstattung oder Bedarfe der technischen Infrastruktur analysiert werden (Arlt/Blum/Gruhler et al. 2010: 33).

${ }^{8}$ Vgl. http://www.zensus2011.de (18.03.2014).

${ }^{9}$ Die Register müssen aus Datenschutzgründen nach der Auswertung, spätestens aber nach vier Jahren wieder gelöscht werden (Gesetz über den registergestützten Zensus im Jahre 2011).
} 
Daneben geben die Landesvermessungseinrichtungen der Bundesländer digitale und analoge topographische Karten sowie historische topographische Karten im Maßstab 1:25.000 bis 1: 100.000 heraus, die die Siedlungsstruktur visuell abbilden. ${ }^{10}$ Eine sehr gute Grundlage für die Abbildung von Siedlungsstrukturen bietet die „Automatisierte Liegenschaftskarte“ (ALK), welche die Gebäudegrundrisse und Flurstücke enthält. Die Gebäude des Liegenschaftskatasters werden zusammengeführt und münden im Produkt „amtliche Hausumringe“. In Verbindung mit den Gebäudehöhen aus dem Liegenschaftskataster ist auch die dritte Dimension der Siedlungsstrukturen beschreibbar. Diese Daten sind nur für Bundesbehörden kostenfrei nutzbar und für größere Gebiete kostenintensiv.

\subsection{Kommunalstatistiken}

Viele statistische Ämter der Kommunen haben ihre eigenen Raumbezüge unterhalb der Gemeindeebene, z. B. Stadtteile, statistische Bezirke oder Unterbezirke und Baublöcke, auf denen sie kleinräumige Daten bereitstellen. Der Verband der Deutschen Städtestatistiker hat einen Leitfaden erarbeitet, der eine eindeutige EDV-gestützte Zuordnung statistischer Daten ermöglichen soll (Verband Deutscher Städtestatistiker 1991). Die Arbeitsgemeinschaft Kommunalstatistik KOSTAT, die vom Bundesinstitut für Bau-, Stadt- und Raumforschung und den statistischen Ämtern mehrerer Großstädte gebildet wird, arbeitet an kommunalstatistischen kleinräumig gegliederten Daten. 100 Städte Deutschlands, darunter fast alle Großstädte, sind an dem Projekt beteiligt und verfügen über kleinräumige Einwohnerdaten und teilweise Gebäudedaten. ${ }^{11}$ Baulich-räumliche Daten liegen nur wenigen Kommunen vor und weisen lediglich bestimmte Bauperioden und beispielsweise die Bebauungsform Ein-/ Mehrfamilienhaus auf der Stadtteilebene aus. Kleinräumige, inhaltlich differenzierte baulich-räumliche Daten, die auch regelmäßig fortgeführt werden, liegen den Kommunen in der Regel nicht vor.

\subsection{Kommerzielle Geofachdaten}

Privatwirtschaftliche Anbieter besitzen eine Vielzahl von Geofachdaten. Infas 360 stellt „hochdifferenzierte“ Gebäudetypen, die durch umfassende Hausbegehungen erhoben wurden, und Gebäudegrundflächen zur Verfügung. Es können „feinräumig homogene Quartiere“ gleicher Bebauung abgebildet werden. Diese Gebäudedaten sind bundesweit erhältlich. ${ }^{12}$ Die Erstellung der Daten ist jedoch nicht trans-

\footnotetext{
${ }^{10} \mathrm{Vgl}$. http://www.bkg.bund.de (18.03.2014).

${ }^{11}$ Vgl. http://www.staedtestatistik.de (18.03.2014).

${ }^{12} \mathrm{Vgl}$. http://infas360.de/kundendaten/mikrodatenkatalog/ und http:// infas360.de/geodaten/geostrukturen/ (09.04.2014).
}

parent, und die Daten wären für großräumige Untersuchungen sehr teuer. Der hohe Preis zeigt den hohen Wert der Daten, die bisher vorwiegend zur Optimierung von Wirtschafts- und Verkaufskonzepten genutzt werden (Meinel/ Hecht/Herold et al. 2008: 62; Hecht/Herold/Meinel 2010: 217; Hecht 2013: 285). Privatwirtschaftliche Beratungsunternehmen wie „empirica“ und „microm“ haben ebenfalls eine große Mengen an kleinräumigen, gebäudebezogenen Geodaten oder Geofachdaten, die im Rahmen von Auftragsforschungen für Bund, Kommunen, aber auch Banken und Bauträger genutzt, aber nicht als Datensatz angeboten werden.

\subsection{Settlement Analyzer $\left(\right.$ SEMENTA $\left.^{\circledR}\right)$}

Am Leibniz-Institut für ökologische Raumentwicklung (IÖR) wurde das GIS-basierte Werkzeug SEMENTA ${ }^{\circledR}$ entwickelt, mit dem eine Analyse der Siedlungsstruktur möglich wird (vgl. Meinel/Hecht/Herold et al. 2008). Es füllt die Lücke zwischen den nur groben amtlichen Daten und den detaillierten kartographischen Darstellungen der Siedlungsstruktur, indem die visuellen kartographischen Daten für quantitative Analysen verwertbar gemacht werden. Mittels SEMENTA ${ }^{\circledR}$ werden auf der Grundlage von (digitalen) topographischen Karten im Maßstab 1:25.000 Gebäude automatisiert klassifiziert. Es werden dabei acht Gebäudetypen mit Wohnnutzung und zwei Gebäudetypen mit Nichtwohnnutzung unterschieden (vgl. Tab. 2). Die Gebäudetypen werden mit hoher Genauigkeit ermittelt und gebäudescharf bestimmt. Erst in einem nächsten Schritt werden die Daten auf ATKIS-Grenzen aggregiert (Hecht/ Herold/Meinel 2010: 224). Das Verfahren des zusätzlichen Werkzeugs SEMENTA ${ }^{\circledR}$-CHANGE besteht darin, analoge topographische Karten im Maßstab 1:25.000 in GIS einzulesen, die Gebäude automatisiert zu extrahieren ${ }^{13}$ und in einem zweiten Schritt zu klassifizieren. In der Überlagerung von Karten unterschiedlicher Zeitstände können neue oder abgerissene Gebäude und damit Veränderungen der Siedlungsstruktur erkannt werden (Hecht/Herold/Meinel 2010: 219 ff.).

Die ermittelten Gebäudetypen können mit weiteren Daten angereichert werden, beispielsweise mit der Geschosszahl und Wohnungszahl der Wohngebäude aus regional verfügbaren Referenzwerten. Daraus lassen sich Kennzahlen wie

\footnotetext{
${ }^{13}$ Die Grenzen von SEMENTA ${ }^{\circledR}$-CHANGE liegen darin, dass topographische Karten bis vor wenigen Jahren analog hergestellt wurden und sich z. B. bei dicht bebauten Gebieten durch Schraffuren oder durch Staffage Ungenauigkeiten ergeben, die die Erkennung von Neubauten und Abrissen erschweren. Mit den „Digitalen Topographischen Karten" in neuer Kartengraphik (DTK 25) ist der aufwendige Schritt der Gebäudeextraktion nicht mehr erforderlich, da hier die Gebäude auf einer separaten Ebene verfügbar sind (Hecht/Herold/Meinel 2010: 219).
} 
Tab. 2 Gebäudetypologie aus SEMENTA ${ }^{\circledR}$. (Quelle: Vereinfacht nach Meinel/Hecht/Herold et al. (2008: 34))

\begin{tabular}{ll}
\hline MFH in Blockstruktur & $\begin{array}{l}\text { Mehrfamilienhaus traditionell in geschlos- } \\
\text { sener Bauweise } \\
\end{array}$ \\
& $\begin{array}{l}\text { Mehrfamilienhaus (traditionell oder neu) } \\
\text { freistehend }\end{array}$ \\
& Mehrfamilienhaus traditionell in Zeile \\
MFH in offener & Mehrfamilienhaus industriell in Zeile \\
Blockstruktur & Hochhaus ( $>50 \mathrm{~m}$ ) \\
Ein- und & Ein- und Zweifamilienhaus \\
Zweifamilienhäuser & $\begin{array}{l}\text { Reihenhaus } \\
\text { Dörflich traditionelles Haus }\end{array}$ \\
Nichtwohnnutzung & $\begin{array}{l}\text { Industrie/Gewerbe } \\
\text { Besondere funktionale Prägung (z. B. } \\
\text { Verwaltung, Gesundheit/Soziales, Bildung/ }\end{array}$ \\
& Forschung, Kultur) \\
\hline
\end{tabular}

${ }^{a}$ Eine weitere Unterscheidung der Nichtwohnnutzung ist geplant

die Grundflächen(-dichte), die Geschossflächen(-dichte) und Einwohnerdichten berechnen (Meinel/Hecht/Herold et al. 2008: 40). Dabei ist allerdings zu beachten, dass die Aussagekraft der Kennzahlen durch die Anreicherung mit Referenzwerten gemindert wird und diese nur für regionale und vergleichende Untersuchungen verwendet werden sollten (Hecht/Herold/Meinel 2010: 230).

Grundsätzlich besteht mit diesen quantitativ verfügbaren baulich-räumlichen Daten aus SEMENTA ${ }^{\circledR}$ die Möglichkeit, Analysen für große Gebietskulissen (z. B. Städte, Regionen, deutschlandweit), kleinräumig (z. B. gebäudescharf, statistische Baublöcke, 100m-Raster), und für Zeitreihen (z. B. retrospektiv, zukünftig in jährlicher Fortschreibung) durchzuführen. Die Daten werden bisher im Rahmen von Forschungsprojekten und Auftragsstudien verwendet.

\subsection{Anforderungen an baulich-räumliche Daten}

Kleinräumige baulich-räumliche Daten liegen derzeit nur wenigen Kommunen, kommerziellen Anbietern von Geofachdaten und einer geringen Zahl von Beratungsunternehmen bzw. Forschungsinstituten vor und weisen unterschiedliche Qualitätsmerkmale auf. Wünschenswert wäre eine Plattform oder Datenquelle, in der baulich-räumliche Daten nach den drei folgenden Anforderungen bereitgestellt werden:

\section{Inhaltlich sollten baulich-räumliche Daten als Indikatoren den Gebäudetyp und die Bauperiode aufweisen, zudem die Gebäudegrundflächen(-dichte), Geschosszahlen und Geschossflächen(-dichte)}

In Analysen des Wohnungsbestandes haben sich der Gebäudetyp und die Bauperiode als wichtiges Unterscheidungsmerkmal herausgestellt. Beide spielen eine wichtige Rolle bei der Einschätzung von Grundrissen und Ausstattungs- merkmalen, Materialität, Gebäudehöhe und Geschosszahl, Wohnumfeld, Erschließungssystem, Eigentümerstruktur etc. und geben bei den jüngeren (noch unsanierten) Wohnungsbeständen auch Hinweise auf den baulichen Zustand. Die bisherige Unterscheidung der amtlichen Daten aus dem Zensus 2011 in „gereihtes Haus, Doppelhaushälfte“ etc. lässt den Geschosswohnungsbau weitgehend undifferenziert und ist für viele Fragestellungen nicht ausreichend. Eine sinnvolle Unterteilung der Gebäudetypen nach Bauperioden, die auch Hinweise auf die genannten qualitativen Merkmale gibt und sich aus bisherigen Forschungsarbeiten (insbesondere Blum/Gruhler 2010) ableiten lässt, wäre beispielsweise eine Unterscheidung in Villen und Blockrandbebauungen der Gründerzeit, Zeilenbauten der 1920er/1930er und 1950er/1960er Jahre, Plattenbauten der 1970er/1980er Jahre und Einfamilienhäuser der 1950er bis 1970er Jahre. Für stadtmorphologische und stadtplanerische Analysen ist zudem die Gebäudegeometrie erforderlich, das heißt die Informationen zu Gebäudegrundflächen, Gebäudehöhe, Geschosszahlen und Geschossflächen sowie die dazugehörigen Dichtewerte. Für die Bestandsentwicklung wären des Weiteren Daten zum baulichen Zustand bzw. Veränderungen sehr hilfreich, um das Ausmaß bzw. das Erneuerungsvolumen und Auswirkungen von baulichen Veränderungen abschätzen zu können (vgl. Koll-Schretzenmayr/Kramp 2010). ${ }^{14}$ Vermutlich werden hier weiterhin qualitative Befragungen von Eigentümern, insbesondere Wohnungsunternehmen, die wichtigste Datenquelle bleiben.

\section{Die Daten sollten kleinräumig verfügbar sein und flexible Raumbezüge aufweisen}

Die deutschlandweit kleinsten einheitlichen statistischen Raumbezüge sind die der Gemeindeebene. Auf der kleinräumigen Ebene unterhalb der Gemeindegrenzen fehlen kongruente, ortsstabile Raumbezüge und flächendeckende Daten. ${ }^{15}$ Auf regionaler Ebene würde man daher erhebliche Schwierigkeiten haben, eine Vielzahl von kleinräumigen Daten mit ihren unterschiedlichen Raumbezügen zu vereinen und zu überlagern. Die baulich-räumlichen Daten sollten daher flexibel in die Monitoring-Systeme von Kommunen integrierbar sein. Alternativ wäre eine deutschlandweite Darstellung, z. B. über ein kleinräumiges 100-m-Raster, denkbar, die unabhängig von den Raumbezügen der Kommunen ist. Solche Rasterdarstellungen werden zunehmend verwendet, erfordern aber auch neue Sehgewohnheiten. Gebäudescharfe Informationen sind wiederum sinnvoll,

\footnotetext{
${ }^{14}$ Es wird versucht, die anonymisierten Registerdaten des Zensus 2011 zur Kalibrierung von Schätzverfahren (wie SEMENTA ${ }^{\circledR}$ ) zu nutzen, bevor diese gelöscht werden.

${ }^{15}$ ATKIS-Baublöcke, die auf naturräumlichen und baulichen Grenzen basieren, sind deutschlandweit vorhanden, werden aber von den Kommunen nicht für statistische Berechnungen genutzt.
} 
wenn Möglichkeiten und Maßnahmen beispielsweise zur Innenentwicklung bewertet werden sollen.

Die Daten sollten in ihrer Herstellung transparent, die Fortschreibung der Daten gesichert und die Daten für Kommunen und Wissenschaft, erschwinglich' sein

Eine transparente Herstellung und eine zukünftig gesicherte Fortschreibung der Daten sind wichtigste Kriterien für die Verwendung in der Planung. Grundsätzlich sollten die Daten für kommunale und wissenschaftliche Forschungszwecke zudem ,erschwinglich' sein. Denkbar wäre eine Bereitstellung der Daten über Geo-Portale auf kommunaler Ebene oder Landesebene im Rahmen der „Geodateninfrastruktur Deutschland“" die über die EU-Richtlinie INSPIRE umgesetzt wird. ${ }^{16}$

\section{Nutzen und Mehrwert baulich-räumlicher Daten}

\subsection{Nutzen kleinräumiger Siedlungsanalysen}

Die aufgezeigten Forschungsarbeiten sind Einzelstudien, die jeweils Anwendungsbereiche baulich-räumlicher Daten für die Planung darstellen, aber einen verallgemeinerbaren Nutzen erkennen lassen. Im Folgenden soll gezeigt werden, dass eine Einbindung von baulich-räumlichen Daten in die Monitoring-Systeme von Kommunen sinnvoll und hilfreich ist. Bei einem baulich-räumlich basierten Monitoring lassen sich drei Analyseschritte unterscheiden, die möglicherweise von unterschiedlichen Akteuren vorgenommen werden. Der erste Schritt ist das Abbilden bzw. Monitoring, bei dem Eigenschaften, Regelmäßigkeiten und Veränderungen von Siedlungsstrukturen beschrieben, baulich-räumliche Daten beispielsweise mit sozialen Daten verknüpft, komplexitätsreduzierende Typisierungen vorgenommen und die Ergebnisse in Karten visualisiert werden. Als Analysen im Rahmen von Monitoring-Systemen ergibt sich eine Vielzahl von Potenzialen:

- Quantifizierung der Siedlungsflächenentwicklung, Entdeckung von Mustern und Ausmaßen baulichräumlicher Merkmale sowie die Veränderungen der Siedlungsstrukturen,

- Identifikation und überschlägige Berechnungen der Innenentwicklungspotenziale, auch unter Verwendung der baulichen Dichte des Umfeldes,

- Ermittlung von städtischen, suburbanen oder auch dörflichen Siedlungstypen, z. B. zu bestimmten Fragestellungen der Wohnungsmarktlage und Nachfragegruppen,

\footnotetext{
${ }^{16}$ Vgl. http://www.geoportal.de (18.03.2014).
}

Verdichtungs- und Freiflächenpotenzialen, energetischem Sanierungsbedarf,

- Identifikation von Veränderungen wie Generationenwechsel, Sozialstruktur und Nachfragerückgang durch gebäudetypbasierte Differenzierungen von Veränderungsprozessen, z. B. durch Verknüpfung der Gebäudetypen mit demographischen und sozioökonomischen Daten,

- Identifikation und überschlägige kleinräumige Berechnungen der Bevölkerungsverteilung auf der Grundlage von Stadtstrukturtypen, z. B. für Bedarfsanalysen zur sozialen Infrastruktur, zu Verkehr, Leitungsinfrastruktur, Energie, Abfall (insbesondere wenn kleinräumige Bevölkerungsdaten nicht verfügbar sind, wie z. B. in ländlichen Räumen),

- Identifikation von Gebäudetypen, bei denen bestimmte bauliche Maßnahmen besonders wirkungsvolle soziale Auswirkungen haben können, Verwendung dieses Wissens für gebäudetypbasierte Zielvorgaben und die Kommunikation mit der Wohnungswirtschaft.

Der zweite Schritt ist der des Verstehens und Interpretierens der Ergebnisse, das heißt eine Analyse der Wirkungszusammenhänge und eine Klärung der Einflussmöglichkeiten. Hier sind vertiefende quantitative Analysen der Daten und die Analysen von qualitativen Informationen aus Experteninterviews, Vor-Ort-Begehungen etc. unerlässlich. Die beiden vorigen Analyseschritte und Ergebnisse münden im Bewerten und Entscheiden, das heißt, dass Beobachtungen hinsichtlich der stadtpolitischen, strategischen und räumlichen Zielsetzungen bewertet, Zielvorgaben entwickelt und Maßnahmen umgesetzt werden. Beispiele dafür sind:

- Evaluierung von Maßnahmen der Innenentwicklung versus Außenentwicklung und Verdichtung sowie der Freiraumentwicklung,

- Evaluierung von Maßnahmen für einen Gebäudebestand einer ausgewählten Bauperiode, z. B. hinsichtlich baulicher und energetischer Aufwertung, bestimmter Bewohner- und Nachfragegruppen, preisgünstigem Wohnraum,

- Evaluierung der Wirkung von Stadtplanungspolitik und Städtebauförderung, z. B. bei Förderung baulicher Maßnahmen (bauliche und energetische Aufwertung von Gebäuden, Wohnungszusammenlegungen, Baulandvergabe).

\subsection{Mehrwert baulich-räumlicher Daten}

Ein zusätzlicher Datensatz im Monitoringsystem einer Kommune oder eines Regionalverbands verursacht einen erheblichen Aufwand, wodurch die Frage aufkommen mag, ob die baulich-räumlichen Daten nicht durch andere quantitative oder qualitative Daten substituiert werden könnten. Hierzu sollen abschließend für den Mehrwert vier Aspekte 
hervorgehoben werden: die Visualisierung, die kleinräumige Analyse der baulich-räumlichen Siedlungsstruktur, die Verknüpfung anderer Daten mit der baulich-räumlichen Struktur (als Grundlage für bauliche Maßnahmen) und die Grundlage für überschlägige Berechnungen mittels Stadtstrukturtypen.

Die ,verortete' Visualisierung statistischer Kennwerte stellt einen erheblichen Mehrwert für Planer selbst, aber auch als Kommunikationsmittel mit Politikern, Akteuren der Fachämter, der Wohnungswirtschaft und auch der Öffentlichkeit dar. Bei qualitativen Analysen zum Gebäudebestand ist dies nicht möglich.

Die kleinräumige Analyse der baulich-räumlichen Siedlungsstruktur aus Karten erlaubt es, baulich-räumliche Strukturen, Muster und Veränderungen quantitativ zu analysieren. Der Mehrwert liegt in Verbindung mit GIS darin, dass eine quantitative Auswertung der Daten anstatt einer qualitativen (visuellen) Interpretation von Karten möglich wird. Dies erscheint bei einer Verlagerung von der wachsenden zur bestandsorientierten Siedlungsentwicklung unumgänglich.

Insbesondere der Verknüpfung von baulich-räumlichen Daten mit anderen (z. B. sozialen) Daten wird ein hoher Mehrwert beigemessen, da sich daraus Handlungsempfehlungen für bestimmte Gebäudetypen ableiten lassen. Eine gebäudescharfe Erkennung von Gebäudetypen und Bauperioden ermöglicht gebäudetypbasierte Analysen, die bisher nicht möglich waren und durch ihre Kleinräumigkeit (z. B. auf Baublockebene oder Wohnblockebene) und Übertragbarkeit eine sehr viel höhere Aussagekraft haben, als Analysen von Stadtteilen, die von einem bestimmten Gebäudetyp oder einer Bauperiode dominiert werden. Ein rein sozialräumliches Monitoring bringt zwar Aussagen beispielsweise hinsichtlich Infrastrukturbedarfen und sozialen Problemlagen, lässt aber keine Schlüsse zu baulichen Maßnahmen im Wohnungsbestand $\mathrm{zu}$.

Des Weiteren lassen sich baulich-räumliche Daten zu Gebäudetypen bzw. Stadtstrukturtypen für überschlägige Berechnungen verwenden, um gebäudetypspezifische Einwohnerdichten anzunehmen und daraus Aussagen zur sozialen Infrastruktur, dem Öffentlichen Personennahverkehr (ÖPNV) etc. abzuleiten. Dies stellt einen wichtigen Mehrwert dar, wenn keine anderen kleinräumigen Daten verfügbar sind, wie z. B. im ländlichen Raum.

Diese vier Aspekte werden als enormes Potenzial für eine bestandsorientierte Siedlungsentwicklung und ein intelligentes Management von Städten und Regionen gesehen, das weder durch andere quantitative Daten noch mit qualitativen Studien möglich ist.

\subsection{Fazit}

Eine bestandsorientierte Siedlungsentwicklung erfordert eine ausgewogene Betrachtung der Stadt hinsichtlich ihrer sozialen, ökologischen und wirtschaftlichen und nicht zuletzt ihrer baulich-räumlichen Struktur und Entwicklung. Bisher bilden soziale und funktionale Merkmale einen Schwerpunkt in Monitoring-Systemen. Beim Aufbau dieser Systeme sollten zukünftig auch baulich-räumliche Merkmale stärker berücksichtigt werden, um im Sinne einer strategischen Stadtentwicklung Zielvorgaben und Handlungsempfehlungen für die gebaute Umwelt abzuleiten. Um kleinräumige Siedlungsanalysen durchzuführen, ist jedoch eine starke Verbesserung einer ,erschwinglichen " und transparent erstellten baulich-räumlichen Datenlage für die Kommunen erforderlich, sei es durch die amtliche Statistik oder durch andere Geodatenanbieter. Der Beitrag ist damit ein Anstoß für eine Verbesserung der Datenlage und ein Plädoyer für kleinräumige Siedlungsanalysen.

Danksagung Ich danke Prof. Dr. Barbara Zibell für ihre kritischen Hinweise und Anregungen zum Manuskript. Mein Dank geht zudem an Dr. Gotthard Meinel, Dr. Robert Hecht und Hendrik Herold für ihre Unterstützung bei der Verwendung von SEMENTA ${ }^{\circledR}$ und die zahlreichen anregenden Fachdiskussionen.

\section{Literatur}

Albers, G. (2007): Strukturmodelle für die Stadtentwicklung - gerichtet auf Wachstumslenkung, geeignet für Schrumpfungslenkung? In: Giseke, U.; Spiegel, E. (Hrsg.): Stadtlichtungen: Irritationen, Perspektiven, Strategien. Gütersloh/Berlin, 31-46. = BauweltFundamente 138.

Amt für Städtebau Zürich (2012): Dichter: Eine Dokumentation der baulichen Veränderung in Zürich - 30 Beispiele. Zürich.

Arlt, G.; Blum, A.; Gruhler, K.; Lehmann, I. (2010): Siedlungsraumbezogene Strukturtypen. In: Blum, A.; Gruhler, K. (Hrsg.): Typologien der gebauten Umwelt. Modellierung und Analyse der Siedlungsentwicklung mit dem Strukturtypenansatz. Aachen, $27-38$.

Banse, J.; Effenberger, K.-H. (2010): Strukturtypen der Wohnbebauung als Basis für Wohnungsmarktanalysen. In: Blum, A.; Gruhler, K. (Hrsg.): Typologien der gebauten Umwelt. Modellierung und Analyse der Siedlungsentwicklung mit dem Strukturtypenansatz. Aachen, 93-116.

BBSR - Bundesinstitut für Bau-, Stadt- und Raumforschung (2011): Umsetzung von Maßnahmen zur Reduzierung der Flächeninanspruchnahme: Innenentwicklungspotenziale. Bonn. http://www. bbsr.bund.de/BBSR/DE/FP/ReFo/Staedtebau/2011/UmsetzungInnenentwicklungspotentiale/01_Start.html?docId=438506\&;notFirst=true (18.03.2014).

Bizer, K.; Ewen, C.; Knieling, J.; Stieß, I. (2009): Zukunftsvorsorge in Stadtquartieren durch Nutzungszyklus-Management: Qualitäten entwickeln und Flächen sparen in Stadt und Region. Detmold.

Bizer, K.; Ewen, C.; Knieling, J.; Stieß, I. (2010): Nachfrageorientiertes Nutzungszyklus-Management: Konzeptionelle Überlegungen für ein nachhaltiges Flächenmanagement in Stadt und Region. Detmold. 
Blum, A.; Gruhler, K. (Hrsg.) (2010): Typologien der gebauten Umwelt. Modellierung und Analyse der Siedlungsentwicklung mit dem Strukturtypenansatz. Aachen.

BMVBS - Bundesministerium für Verkehr, Bau und Stadtentwicklung; BBSR - Bundesinstitut für Bau-, Stadt- und Raumforschung (Hrsg.) (2009): Strategien für Wohnstandorte an der Peripherie der Städte und in Umlandgemeinden. Berlin. = BBSR-Online-Publikation 38/2009. http://www.bbsr.bund.de/BBSR/DE/ Veroeffentlichungen/BBSROnline/2009/DL_ON382009.pdf? blob $=$ publicationFile $\& v=2(18.03 .2014)$.

Bundesministerium für Raumordnung, Bauwesen und Städtebau (1986): Baulandbericht 1986. Bericht des Bundesministers für Raumordnung, Bauwesen und Städtebau. Bonn.

Bundesregierung (2002): Perspektiven für Deutschland: Unsere Strategie für eine nachhaltige Entwicklung. Berlin.

Conzen, M. R. G. (1960): Alnwick, Northumberland: a study in townplan analysis. London.

Conzen, M. P. (Hrsg.) (2004): Thinking about Urban Form. Papers on Urban Morphology 1932-1998, M. R. G. Conzen. Oxford.

Curdes, G. (1989): Zum stadtmorphologischen Ansatz der ,italienischen Schule“. In: Curdes, G.; Haase, A.; Rodriguez-Lores, J. (Hrsg.): Stadtstruktur: Stabilität und Wandel. Beiträge zur stadtmorphologischen Diskussion. Köln, 143-148. = Politik und Planung 22.

Curdes, G. (1993): Stadtstruktur und Stadtgestaltung. Stuttgart, Berlin.

Curdes, G. (1999): Die Entwicklung des Aachener Stadtraumes. Dortmund.

Curdes, G.; Ulrich, M. (1997): Die Entwicklung des Kölner Stadtraumes. Dortmund.

Diener, R.; Herzog, J.; Meili, M.; de Meuron, P.; Schmid, C. (2006): Die Schweiz. Ein städtebauliches Portrait. Basel.

Dießelmann, M.; Meinel, G.; Hecht, R. (2011): Siedlungsflächenmonitoring in Rasterkarten. In: Meinel, G.; Schumacher, U. (Hrsg.): Flächennutzungsmonitoring III. Erhebung, Analyse, Bewertung. Berlin, 61-70. = IÖR-Schriften 58.

Evans, R. (2005): Urban morphology - Introduction. In: Urban Design 93, 16.

Frick, D. (2011): Theorie des Städtebaus: Zur baulich-räumlichen Organisation von Stadt. Tübingen.

Giseke, U.; Spiegel, E. (2007a): Einleitung. In: Giseke, U.; Spiegel, E. (Hrsg.): Stadtlichtungen: Irritationen, Perspektiven, Strategien. Gütersloh/Berlin, 7-17. = Bauwelt-Fundamente 138.

Giseke, U.; Spiegel, E. (Hrsg.) (2007b): Stadtlichtungen: Irritationen, Perspektiven, Strategien. Gütersloh/Berlin. = Bauwelt-Fundamente 138 .

Häußermann, H.; Dohnke, J.; Seidel-Schulze, A. (2010): Synopse der Monitoringsysteme zur sozialen Stadtentwicklung der Städte im Pilotprojekt „Kleinräumiger Städtevergleich“. http://www.difu.de/ sites/difu.de/files/synopse_monitoring_sozialer_stadtentwicklung. pdf (18.03.2014)

Hecht, R. (2013): Automatische Klassifizierung von Gebäudegrundrissen: Ein Beitrag zur kleinräumigen Beschreibung der Siedlungsstruktur. Dissertation an der Technischen Universität Dresden.

Hecht, R.; Herold, H.; Meinel, G. (2010): Analyse und Visualisierung der Siedlungsentwicklung mit SEMENTA ${ }^{\circledR}$-CHANGE. In: Meinel, G.; Schumacher, U. (Hrsg.): Flächennutzungsmonitoring II. Konzepte Indikatoren - Statistik. Berlin, 217-236. = IÖR-Schriften 52.

Heineberg, H. (2006): Geographische Stadtmorphologie in Deutschland im internationalen und interdisziplinären Rahmen. In: Gans, P.; Priebs, A.; Wehrhahn, R. (Hrsg.): Kulturgeographie der Stadt. Kiel, 1-33. = Kieler Geographische Schriften 111.

Hüchtker, S.; Scholz, B.; Selle, K. (2000): Ausgangspunkte und Fragestellung. In: Hüchtker, S.; Scholz, B.; Selle, K.; Sinning, H.; Sutter-Schurr, H. (Hrsg.): Freiräume, Siedlung, Kooperationen: Forschungsergebnisse, Hinweise für die Praxis, Folgerungen. Dortmund, 37-62. = Arbeits- und Organisationsformen für eine nachhaltige Entwicklung 1.
Jessen, J. (2007): Stadtverdünnung? Wie verändert sich die funktionalräumliche und morphologische Struktur von Städten unter den Bedingungen des Schrumpfens? In: Giseke, U.; Spiegel, E. (Hrsg.): Stadtlichtungen: Irritationen, Perspektiven, Strategien. Gütersloh/ Berlin, 47-62. = Bauwelt-Fundamente 138.

Kazig, R.; Wiegandt, C.-C. (2006): Zur Stellung von Architektur im geographischen Denken und Forschen. In: From Outer Space: Architekturtheorie außerhalb der Disziplin 10 (1). http://www. tu-cottbus.de/theoriederarchitektur/Wolke/deu/Themen/051/ Wiegandt/wiegandt.htm (18.03.2014).

Koll-Schretzenmayr, M.; Kramp, S. (2010): Reurbanisierung und bauliche Dynamik: Neubautätigkeiten und ihre räumlichen und sozioökonomischen Auswirkungen in der Stadt Zürich. In: disP 180, $60-80$.

Koll-Schretzenmayr, M.; Ritterhoff, F.; Siebel, W. (2009): In Quest of the Good Urban Life: Socio-spatial Dynamics and Residential Building Stock Transformation in Zurich. In: Urban Studies 46 (13), 2731-2747

Kühne, J. M. (1983): Wohnungsbau und Siedlungsentwicklung in Dortmund. Duisburg.

Larkham, P. (2005): Understanding urban form? In: Urban Design 93, 24.

Lütke-Daldrup, E. (1990): Bestandsorientierter Städtebau: Möglichkeiten, Auswirkungen und Grenzen der Innenentwicklung. Dortmund.

Meinel, G.; Hecht, R.; Herold, H.; Schiller, G. (2008): Automatische Ableitung von stadtstrukturellen Grundlagendaten und Integration in einem Geographischen Informationssystem. Bonn. Bundesministerium für Verkehr, Bau und Stadtentwicklung und Bundesamt für Bauwesen und Raumordnung. = Forschungen 134.

Moudon, A. V. (1998): The Changing Morphology of Suburban Neighborhoods. Cambridge.

Polivka, J.; Roost, F. (2011): Kerne, Adern und Ränder. Siedlungsund Bebauungsstruktur des Ruhrgebiets. In: Reicher, C.; Kunzmann, K. R.; Polivka, J.; Roost, F.; Utku, Y.; Wegener, M. (Hrsg.): Schichten einer Region: Kartenstücke zur räumlichen Struktur des Ruhrgebiets. Berlin, 38-79.

Reicher, C.; Kunzmann, K. R.; Polivka, J.; Roost, F.; Utku, Y.; Wegener, M. (Hrsg.) (2011): Schichten einer Region: Kartenstücke zur räumlichen Struktur des Ruhrgebiets. Berlin.

Schiller, G. (2010): Kostenbewertung der Anpassung zentraler Abwasserentsorgungssysteme bei Bevölkerungsrückgang. Berlin. = IÖR-Schriften 51.

Siedentop, S. (2010): Innenentwicklung / Außenentwicklung. In: Henckel, D.; von Kuczkowski, K.; Lau, P.; Pahl-Weber, E.; Stellmacher, F. (Hrsg.): Planen - Bauen - Umwelt. Ein Handbuch. Wiesbaden, 235-240.

Vachon, G.; Luka, N.; Lacroix, D. (2004): Complexity and contradiction in the ageing early postwar suburbs of Québec City. In: Stanilov, K.; Scheer, B.C. (Hrsg.): Suburban Form: an international perspective. New York, 38-59.

Verband Deutscher Städtestatistiker (1991): Kommunale Gebietsgliederung: Empfehlungen zur Ordnung des Straßen-/Hausnummernsystems und Gliederung des Gemeindegebiets nach Gemeindeteilen, Blöcken und Blockseiten sowie DV-Organisation. Köln. =DST-Beiträge zur Statistik und Stadtforschung 39.

Whitehand, J. W. (1988): The Changing Face of Cities: A Study of Development Cycles and Urban Form. Oxford.

Whitehand, J. W. (2005): Urban morphology, urban landscape and fringe belts. In: Urban Design 93, 19-21.

Wolfram, M. (2011): Kommunale Geodateninfrastrukturen und soziotechnischer Systemwandel: Entwicklungspfade in Deutschland. In: Meinel, G.; Schumacher, U. (Hrsg.): Flächennutzungsmonitoring III. Erhebung, Analyse, Bewertung. Berlin, 241-253. = IÖRSchriften 58 